\title{
Incorporating a smart classroom 2.0 Speech-Driven PowerPoint System (SDPPT) into university teaching
}

\author{
Chia-Li Debra Chen ${ }^{1}$, Yueh-Hsia Chang ${ }^{2}$, Yu-Ta Chien ${ }^{3}$ Charles Tijus $^{4}$ and Chun-Yen Chang ${ }^{1 *}$
}

\author{
* Correspondence: \\ changcy@ntnu.edu.tw \\ ${ }^{1}$ Science Education Center, National \\ Taiwan Normal University, No. 88, \\ Section 4, Ting-Chou Rd., Wunshan \\ District, Taipei 11677, Taiwan \\ Full list of author information is \\ available at the end of the article
}

\begin{abstract}
This study is an ongoing research which aims to renovate the current science learning environment in Taiwan by establishing a Smart Classroom 2.0 through various innovative smart classroom technologies. In this paper, one of the Smart Classroom 2.0 systems, namely the Speech-Driven PowerPoint (SDPPT) system, will be introduced. The SDPPT system is a novel educational advance, which utilizes automatic voice recognition technology to assist PowerPoint Presentations in university teaching. Before the development of various Smart Classroom 2.0 systems, a baseline study was conducted in order to better understand the needs and learning preferences of university students. Having completed the baseline study, the SDPPT system has been deployed and pilot tested in a university classroom. A total of 46 undergraduate students participated in the pilot testing of the system. Students' general perceptions towards learning in an Information and Communication Technologies-Supported Learning (ICT-SL) environment were analyzed using paired sample t-tests. The results indicated that students' learning motivation and learning effectiveness have increased after experiencing the SDPPT system. In addition, students also revealed that they enjoyed the interactions provided by this new system and anticipated the development of the SDPPT would facilitate better learning in classroom environments.
\end{abstract}

\section{Introduction}

With the rapid advances in modern computer technology over the past decades, computer-assisted instruction (CAI) has been deemed as a powerful instructional method in the secondary classroom (Chang and Lee 2010). The National Science Education Standards proposed by the U.S. National Research Council also stated that students should be required to use a wide range of technologies such as computers and software for data display and analysis when conducting scientific investigations (National Research Council 1996). Moreover, the recent science and life technology curriculum standards in Taiwan specifically indicated that students should be able to use computers to store and retrieve information, produce tables and graphs and make spreadsheet calculations in secondary science education (Ministry of Education 2001, 2004).

In response to this advent of the modern Information and Communication Technologies (ICT), many universities in Taiwan have made conscientious efforts to modernize their classrooms by equipping them with technologies (McAlpine and Gandell 2003). In Taiwanese university classrooms, such implemented technology is often referred to as an

\section{黛 Springer}

(c) 2015 Chen et al.; licensee Springer. This is an Open Access article distributed under the terms of the Creative Commons Attribution License (http://creativecommons.org/licenses/by/4.0), which permits unrestricted use, distribution, and reproduction in any medium, provided the original work is properly credited. 
"e-Station" (Winer and Cooperstock 2002). A typical e-Station classroom has an instructor station equipped with a networked computer and audiovisual devices which allow the instructor to teach using various media including presentation software, DVD players, LCD projectors and the like. Even with the addition of these types of technology, many of the e-Station classrooms still seem inadequate in facilitating teaching and learning. Therefore, this study was initiated to renovate the current university classrooms in Taiwan by establishing a "smart classroom" with innovative ICT systems (Chang and Lee 2010). In addition to the features of an e-Station, the smart classroom incorporates innovative technologies to create a classroom with interactive learning materials.

\section{Smart classroom}

A smart classroom is generally referred to as a traditional classroom with multi-technology and media systems installed. By emphasizing monitoring and coordinating features in infrastructure, the installed technologies are expected to make the classroom environment sensitive to meet the teaching and learning needs. In addition to the term "smart classroom" (Chang and Lee 2010; Mao et al. 2002), different but similar terms are also used to describe this kind of modernized classrooms, such as "intelligent classroom" (Winer and Cooperstock 2002), "smart space" (Zhang et al. 2003), "context-aware ubiquitous learning environment" (Hwang et al. 2008), and "ambient intelligence" (Leonidis et al. 2010; Li et al. 2009). Despite different terms used, with the application of information technologies like wireless networks and multimedia devices, the development of smart classrooms shown in the studies all aims at creating a sensitive and intelligent environment in which various types of teaching and learning activities can be supported unobtrusively and seamlessly.

In the design of smart classroom, various functions have been highlighted to support different classroom activity needs, such as to help keep students' class notes up with instructors' lecture, to capture classroom live experiences, and to automate routine classroom tasks (Shi et al. 2003). Different information communication technologies have been used in the related studies to support those functions and needs, including Web-based technologies (Day and Foley 2006; Suo et al. 2009); wireless and mobile technologies (Hwang et al. 2008; Hwang et al. 2011; Liu et al. 2003); digital whiteboard technologies (Smith et al. 2005); audio and video recognition technologies (Chang and Lee 2010; Shi et al. 2003), and face and gesture recognition technologies (Chang and Lee 2010; Chang et al. 2013; Lin et al. 2013).

As mentioned earlier, the higher education institutions in Taiwan has advocated the increase integration of technology into teaching by equipping technologies and media devices to modernize the traditional classroom learning environments. The initiative of National Taiwan Normal University (NTNU) is striving to construct a smart environment at college level named "Smart Classroom 2.0", which consists of various innovative systems (Chang and Lee 2010). This study aims at examining the impact that one of the Smart Classroom 2.0 systems, namely the Speech-Driven Power Point presentation (SDPPT) system, has on teaching and learning.

\section{Learning environment preference of college students}

According to (Fraser 1998, (p.3)), learning environment encompasses "social, physical, psychological, and pedagogical contexts in which learning occurs and affects student 
achievement and attitudes." Having an effective learning environment has thus become one of the teachers' challenges in helping students engage in and take responsibility for their learning (National Research Council 1996). Studies have suggested that the congruence of preferred-actual perceptions enhances students' learning achievement (Fraser and Fisher 1983a,b) and improves learning attitudes toward the subject (Chang et al. 2006). While considering establishing a future Smart Classroom 2.0 for undergraduate students, it is very essential that a baseline study that examines students' preferred learning environment is conducted beforehand, so that students' learning needs can be better fulfilled which eventually will help them achieve better learning outcomes. In this study, a baseline survey on undergraduate students' needs for technology-integrated systems for classroom learning has been conducted.

\section{Baseline study}

The university-wide survey study, namely e-Station Learning Experience and Expectation Questionnaire was conducted to investigate college students' current e-Station classroom learning experiences and their expectations on future smart classroom (Chien and Chang 2010; Chien and Chang 2012). The purpose of the survey was to provide useful information to the development of the Smart Classroom 2.0 tailored to students' needs and learning environment preferences.

A total of 222 undergraduate students, on average of 20 years old, participated in this survey. The 222 students included 108 (49\%) men and 114 (51\%) women; 113(51\%) natural science majors and 109 (49\%) social science majors. The survey questions, designed by 5-point Likert scale items, were focused on investigating students' current eStation classroom learning experience, to what extent the computer technologies were used in classroom settings and students' expectations on future technology-enabled classrooms. The reliability of the survey was 0.91 .

As shown in Table 1, the results indicated that the most frequently used instructional technologies in the college classrooms was PowerPoint presentations followed by multimedia technology, with $72 \%$ and $50 \%$ of the respondents reported using almost in every class. The least frequently used were video conference and chat room, more than $80 \%$ reported never used in their classes.

The results of our survey, as shown in Table 2, also suggested that $87 \%(56 \%=$ expected; $31 \%=$ highly expected) of the 222 university students held high expectations

Table 1 The use of eStation/educational technology in classroom ( $\mathbf{N}=\mathbf{2 2 2}$ )

\begin{tabular}{|c|c|c|c|c|c|}
\hline Educational technology & Mean(SD) & Never & Seldom & Sometimes & Every class \\
\hline PowerPoint Presentation & $3.40(1.08)$ & $14 \%$ & $3 \%$ & $11 \%$ & $72 \%$ \\
\hline LMS Platform (e.g. moodle, blackboard, etc.) & $2.46(1.16)$ & $29 \%$ & $21 \%$ & $24 \%$ & $26 \%$ \\
\hline Chat Room & $1.29(0.64)$ & $80 \%$ & $13 \%$ & $5 \%$ & $2 \%$ \\
\hline E-Mail & $2.12(1.00)$ & $34 \%$ & $31 \%$ & $24 \%$ & $11 \%$ \\
\hline Internet Connection & $2.84(1.10)$ & $16 \%$ & $21 \%$ & $25 \%$ & $38 \%$ \\
\hline $\begin{array}{l}\text { Multimedia Technology (e.g. movie clips, } \\
\text { animations, graphics, etc.) }\end{array}$ & $3.23(0.95)$ & $9 \%$ & $10 \%$ & $31 \%$ & $50 \%$ \\
\hline $\begin{array}{l}\text { Online Activity (e.g. online teaching, online } \\
\text { discussion, online information searching, etc.) }\end{array}$ & $2.08(1.18)$ & $46 \%$ & $18 \%$ & $17 \%$ & $19 \%$ \\
\hline Video Conference & $1.17(0.57)$ & $90 \%$ & $6 \%$ & $2 \%$ & $2 \%$ \\
\hline
\end{tabular}


Table 2 The expectation of technology-enabled smart classroom system development ( $N=222)$

Smart system
Automatic Face Recognition System:
Teachers are able to know each student's
name and classroom learning situations
through the facial characters identified/
captured on the screen at the lecture
podium.

Digital Archive of Course Content: Students 4.47 are able to browse, download, and retrieve data from recorded class sessions, including notes and illustrations that the teacher make on the PPT/electronic whiteboard in class.

\section{Double/Multiple Screen Projection:}

Different teaching materials or student group works are able to be displayed simultaneously with double/multiple screen projection.

Instant Communication System: Through mobile devices, students are able to send instant messages to teacher at the lecture podium to raise or answer questions.

\section{Automatic Voice Recognition System:} Relevant information including documents, pictures, and video clips can be instantly retrieved and shown on the screen when specific keywords and terms are spoken.

Classroom Exceptions Detection System: Through gesture detection shown on the screen at the lecture podium, the teacher is able to know students' classroom learning behaviors.

3D Virtual Reality/Animation-Based Learning Environment: The classroom is surrounded with 3D projection technologies to create simulative and immersive virtual reality for teaching and learning.

Instant Scanning/Projection System: Group works or materials can be scanned/projected instantly and shared with the whole class.

Class Activity Database System: Teacher and students are able to review certain class teaching/learning episodes and group activities to adjust teaching/learning

Interactive Whiteboard System: Students are able to write down answers to teacher's questions or group work results on the electronic tablet device to share with the whole class instantly on the electronic whiteboard.

Class Content Retrieval System: Through voice- or text-based recognition, students are able to search for needed videos/text information from class databases.

Automatic Learning Assessment System: $\quad 3.92$ With automatic assessment tools with quizzes (0.80) and answers, students are able to have selfevaluation on what have learned in classes.

Notes:

'represents "strongly not expected" and 5 "strongly expected".

${ }^{2}$ standard deviation.

(0.70) 
toward using automatic voice recognition technology in their learning. The survey results provide baseline information of how the technology-enabled teaching and learning are functioning in current college classrooms and what students' expectations of a future smart classroom are. With this useful baseline information, it helps researchers and computer engineers to develop a future smart classroom more tailored to students' learning needs and provide a learning environment that can ultimately enhance students' learning outcomes. Taking into account the aforementioned high usage rate of PowerPoint Presentations in university classrooms, and students' great expectations for utilizing voice recognition technology in learning, an integrated system prototype of Speech-Driven PowerPoint (SDPPT) has been developed with the attempt to facilitate students' learning.

\section{Conventional vs. Speech-Driven PowerPoint (SDPPT)}

In the conventional way of conducting the piloted class, the class instructor would raise simple questions to help students brainstorm ideas to facilitate better student-teacher interaction in the classroom. Based on students' answers, the instructor would quickly show the corresponding slides on the PowerPoint to help students visualize and comprehend the concepts presented more easily. However, since students' answers were dependent on the learners' own ideas, the instructor had no way of predicting the sequence of students' answers when preparing the PowerPoint slides. Therefore, based on each answer, the instructor would need to quickly browse through the entire PowerPoint file to find the matching slides. Though this way of conducting the class was applied to improve students' engagement in the class, there existed the tradeoff of interrupting the flow of the class for the purpose of visual presentation.

The newly developed SDPPT system utilized voice recognition technology to identify certain keywords as they were spoken and the system then automatically responded by presenting the corresponding PowerPoint slides on the overhead screen. With the development of the SDPPT system, the instructor could directly call out any desirable PowerPoint slide through a mini and portable handheld microphone. This freed the instructor physically from the teaching platform, and also saved the instructor the time of browsing through the entire PowerPoint file to find the matching slides. Figure 1 illustrates how the SDPPT was used in the classroom.

Voice recognition technology has long been widely utilized in commercial applications, such as mobile phones, for example in the uses of name dialing, phone book searching and vocabulary dictating (Varga and Kiss 2008). Despite the common use of voice recognition technology in the mobile phone industry, its use as an educational tool for retrieving desirable PowerPoint slides in classrooms is a pioneering teaching approach. In the current study, we pilot tested the SDPPT system and examined students' perceptions toward learning in an ICT-supported environment. Students' perceptions in the following three aspects were surveyed: learning motivation with ICT, learning effectiveness with ICT and learning with SDPPT.

\section{Methods}

\section{Participants and procedures}

The Speech-Driven PowerPoint (SDPPT) system was deployed and pilot tested in an elective earth science course. A total of 46 undergraduate students, 22 males and 24 
(A)

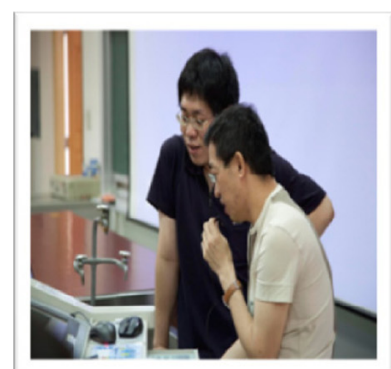

(B)

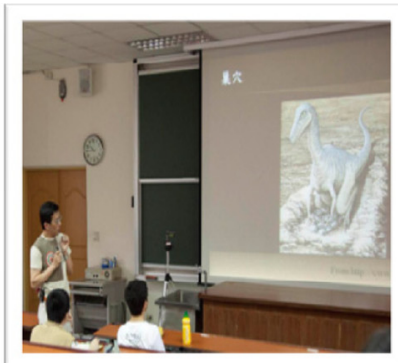

(C)

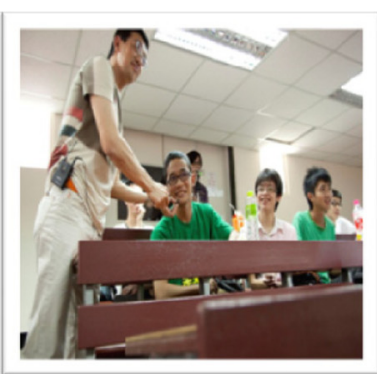

Figure 1 Utilizing the SDPPT in the classroom. (A) SDPPT system testing; (B) SDPPT teaching; (C) Student operating the SDPPT.

female, whose ages ranged from 18 to 24 participated in the study. A week before deploying the system, students were pre-tested for their general perceptions towards learning in an ICT-supported environment. During class time, The SDPPT system was deployed during the lecture. Students were post-tested at the end of the lecture and asked to write reflections on their experiences with the system. The instructor was also interviewed to find out how his teaching was affected when the SDPPT system was used.

\section{Lecture}

The instructor gave a lecture to students about the distinctive characteristics of various dinosaurs and helped students distinguish between the facts and the fallacies about dinosaurs from the movie clip of Jurassic Park. The instructor pre-designed the lesson with the innovative feature of SDPPT. The PowerPoint was pre-programmed so that when certain keywords were spoken, it would automatically present the corresponding slides.

\section{Instrumentation}

\section{AICT-SL questionnaire}

A five point Likert-type scale ranging from 1, strongly disagree, to 5, strongly agree, was used in the questionnaire, namely Attitudes toward ICT-Supported Learning (AICT-SL). The 15-item questionnaire which consisted of 3 factors, learning motivation with ICT, learning effectiveness with ICT, learning with SDPPT, was previously pilot tested to 114 undergraduate students taking the same course. The estimated reliability of AICT-SL was 0.91 . The suitability of data for factor analysis was assessed and the derived Kaiser-Meyer-Oklin value was 0.88 , exceeding the recommended value of 0.6 (Kaiser 1970, 1974). In addition, the Barlett's Test of Sphericity (Barlett 1954) also reached statistical significance $(p=0.000)$, supporting the factorability of the correlation matrix. The rotated solution using Varimax rotation revealed the presence of four factors, which together explained a total of 80.77 percent of the variance. In particular, factor 1 (learning motivation with ICT) contributed 36.1 percent, factor 2, (learning effectiveness with ICT) 23.8 percent, and factor 3, (learning with SD_PPT) 20.8 percent of the variance. The AICT-SL was again administered on the post-test to the class of 46 students and the reliability was also 0.91 . Due to the small sample size in this study, the statistical significance level was set at $\alpha=0.1$. The magnitude of the differences between the means of the pre- and post-test means were assessed using effect size (ES), 
with 0.20 representing a small ES, 0.50, a medium ES, and 0.80, a large ES (Cohen 1988). Note that ES takes into account the size of the difference between the means, regardless of whether or not it is statistically significant. Several researchers have suggested the inadequacy of merely using the result of statistical significance testing for statistical inference since the achievement of statistical significance may depend upon the sample size (Cohen 1988; Daniel 1998; McLean and Ernest 1998). Researchers argued that it is easier to achieve a statistical significance when the sample size is large rather than small and vice versa. With the small sample size in this study, it is more likely to obtain a statistically insignificant result. Therefore, the results of significant effect sizes that occurred in several findings of this study may represent a possibility of achieving statistical significance when a future study is replicated with a larger sample size. Hence, the findings with no statistical significance yet a significant effect size may still provide useful practical significance to the study.

\section{Student reflections}

Students' opinions on learning in an ICT-supported environment were collected through open-ended questions in student reflections. Students were asked to elaborate on how their learning has been affected after exposure to the SDPPT in the following three aspects: 1 . learning motivation with ICT, 2. learning effectiveness with ICT, and 3. interaction with the teacher and fellow students. The research reported in this manuscript has been performed with the ethical approval from the Ministry of Science and Technology under the reference number of MOST 104-2911-I-003-301.

\section{Teacher interview}

Teacher's responses toward the system were also collected through a 10-minute interview. The interview questions focused the on the changes in teaching approaches that the teacher underwent while implementing the SDPPT system in the classroom.

\section{Results and discussion}

\section{Increased learning motivation and student engagement}

The data were analyzed using paired sample t-test. The results, as shown in Table 3, indicated that students' overall attitudes toward ICT-supported learning improved significantly after having experienced the SDPPT system $(t=-3.26, p=0.002, d=0.48$, approaching medium effect size). Particularly, students' perceptions toward learning motivation with ICT increased substantially $(t=-3.58, p=0.001, d=0.53$, medium effect size). Although students' perceptions toward learning effectiveness with ICT and the system itself did not show significant improvements $(t=-1.56, p=0.13, d=0.23$, small effect size; $t=-0.24, p=0.81, d=0.04$ ), there were still small effect size in their perceptions toward learning effectiveness with ICT, and their attitudes on the system were fairly positive to begin with (Pre-test Mean $=4.08$ ). This indicated that students in general had great susceptibility toward the system.

In students' reflections, a majority of them reported that "the system makes learning more fun" and that their "learning interests have greatly increased". Particularly, students also liked the fact that they could have "more interaction with the teacher" when the system was used. For instance, a student commented, "the system is very interesting and it allows the teacher to actually step out of the teaching platform and interact with students directly". Several others also stated that "because the system enables more 
Table 3 Results of students' general perceptions towards learning with ICT and SDPPT

\begin{tabular}{|c|c|c|c|c|c|c|}
\hline Scales & Pre-Test $M$ & Post-Test $M$ & $S D$ & $t$ & $p$ & $d$ \\
\hline Attitudes toward ICT-Supported Learning (AICT-SL) & 3.62 & 3.94 & 0.66 & -3.26 & $0.002^{* *}$ & 0.48 \\
\hline Learning Motivation with ICT (LM-ICT) & 3.29 & 3.92 & 1.20 & -3.58 & $0.001^{* * *}$ & 0.53 \\
\hline Learning Effectiveness with ICT (LE-ICT) & 3.66 & 3.83 & 0.72 & -1.56 & $0.126^{\mathrm{a}}$ & 0.23 \\
\hline Speech-Driven PPT (SDPPT) & 4.08 & 4.11 & 0.91 & -0.24 & 0.810 & 0.04 \\
\hline
\end{tabular}

interaction between the teacher and students", it made them "more willing to participate and to learn in the class".

In addition, the teacher's perceptions generally paralleled with those of the students. As indicated by the teacher, "the fact that one's voice can activate the desired PowerPoint slides in accordance to the speaker's cues allows not only the instructor but also other students to interact with the course contents. This naturally increases students' in class participations". The teacher also indicated that since his teaching style "involves a great deal of moving around in the classroom," this system can "indeed meet teaching needs" by unrestricting him from the teaching platform and enabling him to "interact with the system and the students at the same time."

\section{Improvements in students' self-perceived learning effectiveness}

Though students' perceptions towards learning effectiveness with ICT did not reach statistical significance, the small effect size may still indicate some practical significance $(t=-1.56, p=0.126, d=0.23)$. As a student reflected, "the new system has made learning more efficient without having to switch back and forth from the PowerPoint full screen presentation to the slide sorter view in order to find the matching slides". Several students also expressed that not only their "incentives for learning" increased, but that they had become "more focused" and "paid more attention" in the class when the SDPPT was implemented. Many others also indicated that the system helped "deepen their memory and make their learning more effective". Likewise, the teacher also suggested that he had noticed "an increase in students' attention in class" when the SDPPT system was used as opposed to the regular PowerPoint system.

\section{Greater system stability to reach better learning and teaching efficiency}

Since the system is still in its preliminary stage of development in the field of education, the accuracy of voice recognition still has room for improvements. Two students pointed out that "sometimes the system's retrieving of the wrong slides or simply not responding to the command makes learning inconvenient". Similarly, the teacher stated that he needed to slow down the pace of his speaking and spoke with extra clarity when the system became unstable. This, therefore, caused "extra pressure and anxiety" in both his preparation and teaching of the class.

Despite these negative feedbacks to the system's instability, most of the students still responded optimistically towards the system by stating that their "learning would be much more efficient if the accuracy of the system could be enhanced". The instructor also anticipated the SDPPT system would become more mature in the near future and would greatly benefit teachers' PowerPoint based instruction. The teacher and students 
have both indicated that they "anticipate the development of the SDPPT system will facilitate better learning".

To sum up, this study pioneers a teaching approach of incorporating the SpeechDriven PowerPoint (SDPPT) system, which utilizes automatic voice recognition technology to assist PowerPoint Presentations, in university teaching. Overall, both the teacher and students attitudes were positive towards the system. Students' perceptions towards ICT-supported learning, particularly learning motivation and learning effectiveness with ICT, improved after experiencing the SDPPT system in the class. Students reported that they anticipate the improvements in the accuracy of the system and are expecting to see enhancement of their learning through the aid of the future model of the SDPPT system.

With these advanced smart classroom systems, practitioners still need to be reminded that technology itself cannot improve teaching and learning. Rather, it is how instructors utilize technology and organize teaching and learning activities into good instructional designs that can truly help improve instructions. Therefore, while integrating technologies into classrooms, it is also important to focus on the instructional and learning needs to develop a future smart classroom that can truly facilitate and enhance teaching and learning as a whole.

\section{Limitation}

Although the data of this study indicated that students' perceptions towards ICTsupported learning improved after experiencing the SDPPT system in the class, the possibility that the improvement was attributed by students' curiosity or novelty effect cannot be eliminated. Since people might have the tendency to have increased interests when trying out new technology, it is therefore reasonable to speculate that students' improved perceptions towards ICT-supported learning may not be entirely attributed to the effectiveness of SDPPT system itself but to novelty effect. Due to the pioneering nature of this research, the SDPPT system was only tried out for exemplary innovative teaching practices in a few university classes. In addition, the study has only used the self-report method to survey students' perceived learning motivation and effectiveness on the use of ICT. However, based on the feedbacks collected from the students and the instructor of the current research, we will be able to improve the system by taking account of the feedbacks and replicate the study in a more longitudinal way. Moreover, in our future studies, in addition to merely using students' self-perceived measurement to survey their affective learning outcomes, we will also adopt more objective assessment through the use of empirical data to examine students' cognitive learning outcomes. Through these efforts, we anticipate that the possibility of novelty effect can be minimized as much as possible in our future replicated studies.

\section{Future studies}

Our research will continue to renovate the current science learning environment by fine-tuning the SDPPT system, developing several other Smart Classroom 2.0 systems and hopefully integrating all the systems into mobile devices. In addition to activating PPT slides with voice prompts, we are hoping to further develop the SDPPT system with voice-activated searching feature which allows the instructor for an instant in- 
class search with voice prompts. This way, when students raise questions, the instructor can respond instantly by providing additional and detailed information with the use of voice-activated searching system.

Moreover, for future studies, we will extend our staged efforts to utilize mobile devices into pre-service teacher education. Mobile devices, such as smart phones and tablet PCs, have received substantial attention from the community of educational technology research in the past decade. This rapidly growing interest is due to the great potential mobile devices in making learning more flexible than ever before. However, the renovation of teacher education, to foster the teachers who are capable of mobileassisted teaching, lags far behind the popularization of mobile devices in schools. Therefore, in continuation of the development of Smart Classroom 2.0 Systems, we will also utilize mobile devices into teacher preparation program. We are making every endeavor to integrate various Smart Classroom 2.0 systems, i.e. automatic face recognition system, instant response systems, augmented reality, automatic assessment and the like, into mobile devices.

Having integrated the various systems into mobile devices, we hope that pre-service teachers will have the convenience of utilizing these innovative systems into their teaching, and their abilities in mobile-assisted teaching can be cultivated. Ultimately, we anticipate establishing a more full-fledged Smart Classroom 2.0 learning environment to facilitate teaching and learning and replicating the present study with a substantially larger sample size in the near future.

\section{Consent}

Written informed consents were obtained from the participants for the publication of this paper and any accompanying images.

Competing interests

The authors declare that they have no competing interests.

\section{Authors' contributions}

CLDC has made substantial contributions to the design of the study, acquisition of data, analysis and interpretation of data, and the drafting and revising of the manuscript. YHC has made substantial contributions to the design of the study, acquisition and interpretation of data, and the drafting of the manuscript. YTC has participated in the design of the study, acquisition, analysis and interpretation of data. CT has collaborated in the research project and has given comments on the manuscripts. CYC has participated in conceiving and designing the study, revising the manuscript, and giving final approval of the version to be published.

\section{Acknowledgement}

This research is partially supported by the "Aim for the Top University Project" of National Taiwan Normal University (NTNU), sponsored by the Ministry of Education, Taiwan, R.O.C. and the "International Research-Intensive Center of Excellence Program" of NTNU and Ministry of Science and Technology, Taiwan, R.O.C. under Grant no. MOST 104-2911-I-003-301.

\section{Author details}

${ }^{1}$ Science Education Center, National Taiwan Normal University, No. 88, Section 4, Ting-Chou Rd., Wunshan District, Taipei 11677, Taiwan. ${ }^{2}$ Graduate Institute of Curriculum and Instruction, Tamkang University, No.151, Yingzhuan Rd. Tamsui Dist., New Taipei City 25137, Taiwan. ${ }^{3}$ Graduate Institute of Science Education, National Taiwan Normal University, No. 88, Section 4, Ting-Chou Rd., Wunshan District, Taipei 11677, Taiwan. ${ }^{4}$ Laboratoire "Cognitions Humaine et Artificielle" - CHArt, Université Paris 8, 2, rue de la Liberté, St Denis, Cedex 02 93526, France.

Received: 17 July 2014 Accepted: 7 January 2015

Published online: 10 March 2015

\section{References}

MS Barlett, A note on the multiplying factors for various chi square approximations. J. R. Stat. Soc. 16(Series B), 296-298 (1954) CY Chang, G Lee, A major e-learning project to renovate science learning environment in Taiwan. Turk Online J Educ Technol 9(1), 7-12 (2010) 
CY Chang, CH Hsiao, J Barufaldi, Preferred-actual learning environment 'spaces' and earth science outcomes in Taiwan. Sci. Educ. 90(3), 420-433 (2006)

CY Chang, YT Chien, CY Chiang, MC Lin, HC Lai, Embodying gesture-based multimedia to improve learning. Br. J. Educ. Technol. 44(1), E5-E9 (2013)

YT Chien, CY Chang, Exploring the feasibility of an online contextualized animation-based questionnaire for educational survey. Br. J. Educ. Technol. 41(5), E104-E109 (2010)

YT Chien, CY Chang, Exploring the impact of animation-based questionnaire on conducting a web-based educational survey and its association with vividness of respondents' visual images. Br. J. Educ. Technol. 43(3), E81-E85 (2012)

J Cohen, Statistical Power Analysis for the Behavioral Sciences, 2nd edn. (Lawrence Erlbaum, Hillsdale, NJ, 1988)

LG Daniel, Statistical significance testing: a historical overview of misuse and misinterpretation with implication for the editorial policies of educational journals. Research in the Schools 5(2), 23-32 (1998)

JA Day, JD Foley, Evaluating a Web lecture intervention in a human-computer interaction course. leee Transactions On Education 49(4), 420-431 (2006)

BJ Fraser, Science Learning Environments: Assessment, Effects and Determinants, in International Handbook of Science Education, ed. by BJ Fraser, KG Tobin (Kluwer Academic Publisher, Dordrecht, The Netherlands, 1998), pp. 527-564

BJ Fraser, DL Fisher, Student achievement as a function of person-environment fit: a regression surface analysis. Br J Educ Psychol. 53, 89-99 (1983a)

BJ Fraser, DL Fisher, Use of actual and preferred classroom environment scales in Person-environment fit research. J Educ Psychol. 75, 303-313 (1983b)

GJ Hwang, CC Tsai, SJH Yang, Criteria, strategies and research issues of context-aware ubiquitous learning. Educ Technol Soc 11(2), 81-91 (2008)

GJ Hwang, PH Wu, HR Ke, An interactive concept map approach to supporting mobile learning activities for natural science courses. Comput. Educ. 57(4), 2272-2280 (2011)

H Kaiser, A second generation Little Jiffy. Psychometrika 35, 401-415 (1970)

H Kaiser, An index of factorial simplicity. Psychometrika 39, 31-36 (1974)

A Leonidis, G Margetis, M Antona, C Stephanidis, ClassMATE: Enabling ambient intelligence in the classroom. World Acad. Sci. Eng. Technol. 66, 594-598 (2010)

X Li, L Feng, LZ Zhou, YC Shi, Learning in an Ambient Intelligent World: Enabling Technologies and Practices. [Article; Proceedings Paper]. leee Transactions on Knowledge and Data Engineering 21(6), 910-924 (2009)

MC Lin, MS Tutwiler, YT Chien, CY Chiang, CY Chang, The use of a gesture-based for teaching multiple intelligences: A pilot study. Br. J. Educ. Technol. 44(5), E113-E138 (2013)

TC Liu, HY Wang, JK Liang, TW Chan, HW Ko, JC Yang, Wireless and mobile technologies to enhance teaching and learning. J. Comput. Assist. Learn. 19(3), 371-382 (2003)

YH Mao, WK Xie, YC Shi, GY Xu, X Xiang, Building the Software Infrastructure for Smart Classroom: From Open Agent Architecture (OAA) to Smart Platform, in Advances in Multimedia Information Processing - Pcm 2002, Proceeding, ed. by YC Chen, LW Chang, CT Hsu, vol. 2532 (Springer-Verlag Berlin, Berlin, 2002), pp. 526-533

L McAlpine, T Gandell, Teaching improvement grants: what they tell us about professors' instructional choices for the use of technology in higher education. Br. J. Educ. Technol. 34(3), 281-293 (2003)

JE McLean, JM Ernest, The role of statistical significance testing in educational research. Research in the Schools 5(2), 15-22 (1998)

Ministry of Education, The 1-9 Grades Science and Life Technology Curriculum Standards (Ministry of Education, Taipei, 2001)

Ministry of Education, The Tentative Earth Science Curriculum Guidelines for Senior High Schools (MOE, Taipei, 2004) National Research Council, National Science Education Standards (National Academy Press, Washington, DC, 1996)

YC Shi, WK Xie, GY Xu, R Shi, E Chen, YH Mao, F Liu, The smart classroom: merging technologies for seamless teleeducation. leee Pervasive Computing 2(2), 47-55 (2003)

HJ Smith, S Higgins, K Wall, J Miller, Interactive whiteboards: boon or bandwagon? A critical review of the literature. J. Comput. Assist. Learn. 21(2), 91-101 (2005)

Y Suo, N Miyata, H Morikawa, T Ishida, YC Shi, Open smart classroom: extensible and scalable learning system in smart space using web service technology. leee Transactions on Knowledge and Data Engineering 21(6), 814-828 (2009)

Varga, I., \& Kiss, I. (2008). Speech Recognition in Mobile Phones. Automatic Speech Recognition on Mobile Devices and over Communication Networks, 301-325. Retrieved from http://dx.doi.org/10.1007/978-1-84800-143-5_14

LR Winer, J Cooperstock, The "intelligent classroom": Changing teaching and learning with an evolving technological environment. Comput. Educ. 38, 253-266 (2002)

DG Zhang, EY Chen, YC Shi, GY Xu, A Kind of Smart Space for Remote Real-Time Interactive Learning Based on Pervasive Computing Mode, in Advances in Web-Based Learning - Icwl 2003, Proceedings, ed. by W Zhou, P Nicholson, B Corbitt, J Fong, vol. 2783 (Springer-Verlag Berlin, Berlin, 2003), pp. 297-307 